\title{
Possible Impact of Spinal Anesthesia and Phenylephrine on Sublingual Microcirculation of Cesarean Delivery Patients
}

\author{
Ronald B. George ${ }^{\mathrm{a}, \mathrm{b}, \mathrm{d}}$, Colin Boyd ${ }^{\mathrm{a}}$, Dolores McKeen ${ }^{\mathrm{a}, \mathrm{b}}$, \\ Islam Saleh $\mathrm{Abdo}^{\mathrm{c}}$, Christian Lehmann ${ }^{\mathrm{b}}$
}

\begin{abstract}
Background: This study was a proof of concept of a novel means to evaluate microcirculatory changes during spinal anesthesia for cesarean delivery. It sought to examine the distributive circulatory effects of spinal anesthesia and evaluate the impact of phenylephrine administration on the microcirculation of these women.
\end{abstract}

Methods: After Research Ethics Board approval, healthy, non-laboring pregnant women with singleton, term pregnancies scheduled for elective cesarean delivery were recruited. Participants were randomly assigned to receive either phenylephrine infusion or phenylephrine bolus. Spinal anesthesia was standardized. A sidestream dark-field (SDF) MicroScan ${ }^{\circledR}$ video microscope was applied to the sublingual mucosa to obtain microcirculation videos in five different visual fields. Videos were made before and after spinal anesthesia. The resultant videos were analyzed randomly and blindly. The mean microvascular flow index (MFI) values were compared before and after spinal anesthesia. The difference in MFI following spinal anesthesia was compared between phenylephrine infusion and bolus groups.

Results: Thirty-two patients were recruited for the study; 22 patients had complete video sets for analysis. Baseline characteristics were similar between the two groups, including preoperative hemodynamics. There were no significant differences between pre- and postspinal MFI. The post-spinal MFI within the infusion group (mean \pm standard deviation: $2.74 \pm 0.21$ ) was not significantly different from the bolus group $(2.56 \pm 0.42, \mathrm{P}=0.22)$.

Conclusion: Despite theoretical physiological implications of spinal anesthesia and phenylephrine on the microcirculation, significant alteration of the MFI was not observed between pre- and post-spinal

Manuscript submitted February 15, 2019, accepted May 13, 2019

aDepartment of Women's and Obstetric Anesthesia, IWK Health Centre, Halifax, NS, Canada

bepartment of Anesthesia, Pain Management, and Perioperative Medicine, Dalhousie University, Halifax, NS, Canada

'Department of Anesthesia, Intensive care and Perioperative Medicine, Masaryk Hospital, Usti nad Labem, Czech Republic

dCorresponding Author: Ronald B. George, Department of Women's \& Obstetric Anesthesia, IWK Health Centre, 5850/5980 University Avenue, PO Box 9700, Halifax, NS, B3K 6R8, Canada. Email: rbgeorge@dal.ca

doi: https://doi.org/10.14740/jocmr3778 anesthesia (within group). Additionally, despite an eight-fold larger phenylephrine dose for continuous infusion prophylaxis used in this group of women, this did not result in a significant alteration of the microcirculation compared to those who received phenylephrine treatment for hypotension (between groups).

Keywords: Spinal anesthesia; Microcirculation; Phenylephrine; Sidestream dark-field imaging; Obstetric anesthesia

\section{Introduction}

Maternal hypotension is the most common side effect of spinal anesthesia and is a result of physiologic changes to the macrocirculation $[1,2]$. Spinal anesthesia causes sympathetic nervous system blockade and reduction of systemic vascular resistance. Maternal hypotension is associated with adverse side effects such as maternal nausea and vomiting and fetal acidosis from reduced placental blood flow (secondary to maternal hypotension). If maternal hypotension is treated and/or prevented with vasopressors, specifically the direct $\alpha$-adrenergic agonist, phenylephrine, fetal outcomes as assessed by umbilical arterial and venous $\mathrm{pH}$ are significantly improved [1, 3-6]. These fetal improvements are likely due to the vasopressor effect on the maternal microcirculation and its subsequent impact on macrocirculation and central hemodynamics $[7,8]$.

The microcirculation, consisting of the smallest vessels of the vasculature, is responsible for delivery of oxygen and regulation of blood pressure. Microcirculation by definition includes capillaries, small arterioles and venules which connect immediately adjacent to capillaries to form the pre- and postcapillary units [9]. Currently, our understanding of changes to the microcirculation in pregnant women receiving spinal anesthesia for cesarean delivery is limited. The impact of spinal anesthesia and phenylephrine on the microcirculation of pregnant women using sidestream dark-field (SDF) technology is yet to be reported. This novel study is the first to analyze the microcirculatory response in patients who receive spinal anesthesia and receive two variations of phenylephrine administration for treatment. Abdo et al stated that SDF imaging is a novel approach to the measurement of microcirculation and has a wide application of usefulness within the clinical field [10].

SDF imaging is a stroboscopic light-emitting diode (LED) ring-based imaging modality allowing microscopic assessment 
of microcirculatory capillary density and perfusion in clinical settings [11-13]. This is achieved by an analysis of the moving cells in the images, which permits the quantitative measurement of red blood cell flow in the capillaries. SDF imaging consists of a light guide surrounded by a $530 \mathrm{~nm}$ LED, a wavelength of light that is absorbed by the hemoglobin of red blood cells, which allows for their observation as dark cells flowing in the microcirculation. It permits quantification of microvascular blood flow using the microvascular flow index (MFI) and vessel density.

The primary objective of this research was to examine the physiologic impact of spinal anesthesia and the influence of prophylactic phenylephrine infusion (PEI) versus phenylephrine bolus (PEB) administration on the distributive circulation. This objective was measured by SDF imaging on the sublingual microcirculation of pregnant subjects. The SDF imaging was performed pre- and post-spinal anesthesia, prior to neonatal delivery during cesarean delivery. We also compared the influence of PEI administered for prophylaxis to PEB administered for treatment of hypotension on sublingual microcirculation following administration of spinal anesthesia. We hypothesized that the combined impact of spinal anesthesia and phenylephrine on the microcirculation would be a reduced MFI, vascular density and proportion of perfused vessels.

\section{Materials and Methods}

With Institutional Research Ethics Board approval (IWK REB \#1010276), ASA physical status II pregnant women with singleton gestation at gestational age $>36$ weeks scheduled for elective cesarean delivery under spinal anesthesia were recruited. This study was conducted in compliance with the ethical standards of the IWK Health Centre on human subjects as well as with the Helsinki Declaration. Patients were recruited at the IWK Health Centre, Halifax, NS, Canada. Exclusion criteria included: women who were in labor, had a body mass index $(\mathrm{BMI})>35 \mathrm{~kg} / \mathrm{m}^{2}$, type 1 diabetes mellitus, hypertensive disease or cardiac disease, an allergy to phenylephrine or any other standardized medication, were smokers, or had any caffeine intake within $6 \mathrm{~h}$ of SDF measurement. Patients were withdrawn if they were unable to complete the SDF imaging or if they had a failed spinal anesthetic (i.e. block lower than T6 dermatome or greater than T2 dermatome). Block height was determined with a blunt tip needle approximately 5 min after spinal anesthetic was administered.

After informed consent was obtained, patients were randomly assigned to one of two groups: PEI or PEB. Patient allocation was performed by opening opaque envelopes containing the patient's group and study ID number prepared by research staff. Randomization was according to a computergenerated randomization order (Random.org). Maternal age, height, weight, BMI and gestational age were recorded. Blood pressure and heart rate were measured in the supine position with left uterine displacement. Baseline systolic blood pressure (SBP) was determined by calculating the mean of three consecutive SBP measurements taken in the preoperative area.

All patients received antacid prophylaxis with $30 \mathrm{~mL}$ so- dium citrate and were transferred to the operative room. Spinal anesthesia was clinically administered at the L3-L4 interspace in the sitting position using $12 \mathrm{mg}$ of hyperbaric bupivacaine with $15 \mu \mathrm{g}$ of fentanyl and $150 \mu \mathrm{g}$ of preservative free morphine. Patients were then immediately laid supine with left uterine displacement to minimize aortocaval compression and hypotension. All patients received a crystalloid fluid co-load immediately after the spinal. This was gravity delivered with a goal of $1,000 \mathrm{~mL}$ prior to delivery. The dermatomal level affected was determined 5 min after spinal anesthesia by the attending anesthesiologist and recorded. Blood pressure was measured at 1-min intervals for the first 15 min immediately after the spinal anesthetic, then every $2.5 \mathrm{~min}$ until the time of delivery. Hypotension was defined as the higher blood pressure of either SBP $<80 \%$ of baseline or $<90 \mathrm{~mm} \mathrm{Hg}$. Hypertension was defined as SBP $>120 \%$ of baseline.

Patients in the PEI group had a PEI of $30 \mu \mathrm{g} / \mathrm{min}$ started immediately after administration of spinal anesthesia in conjunction with crystalloid fluid co-loading. Participants in the PEB group were also given the same crystalloid co-load after the spinal was administered. Those in the PEB group who experienced hypotension (range predefined) were treated with $50-100 \mu \mathrm{g}$ boluses of phenylephrine (5 - $10 \mathrm{mg}$ boluses of ephedrine second line therapy). The number and type of interventions needed to maintain the blood pressure in the patients' target range were documented. The total amount of intravenous fluid administered, as well as the total cumulative dose of vasopressors administered during the study period, was also recorded.

The initial SDF measurements were completed in the obstetric preoperative area with the patient supine with left uterine displacement, mirroring the position in the operating room. The second set of paired post images were then completed 10 min after spinal anesthesia was initiated. Trained research staff recorded all noninvasive SDF images using the Micro$\mathrm{Scan}^{\circledR}$ video microscope (MicroVision Medical, Amsterdam, The Netherlands). The SDF device was equipped with a sterile LED probe cap and was gently applied to the sublingual oral mucosa to avoid pressure artifact. Steady images of at least $10 \mathrm{~s}$ duration were recorded and stored digitally on a research laptop. Digital recordings of five different visual fields were obtained for each participant, with minimal intervals between recordings.

Research staff quantified blood flow using semi-quantitative analysis software (AVA 3, MicroVision Medical) which was developed specifically for the MicroScan ${ }^{\circledR}$ video microscope. Video clips were analyzed by the same, single research staff according to the established guidelines [14]. The video images, which contain no identifiable information, were analyzed at random to prevent coupling between images. An investigator not involved with the video analysis completed the randomization of the videos with a randomization table generated on Random.org separate from the participant allocation randomization. Demographic and clinical data were collected and presented as mean (standard deviation), median (interquartile range), or counts as appropriate using SPSS Statistic 21 software package (IBM Corporation, Armonk, NY, USA).

The primary outcome was MFI pre- and post-spinal anesthesia. Additionally, comparison of the impact of vasopres- 
Table 1. Demographic Characteristics

\begin{tabular}{llll} 
& Phenylephrine infusion $(\mathbf{n}=\mathbf{1 1})$ & Phenylephrine bolus $(\mathbf{n}=\mathbf{1 1})$ & P-value \\
\hline Age (years) & $32 \pm 5$ & $34 \pm 5$ & 0.31 \\
BMI $\left(\mathrm{kg} / \mathrm{m}^{2}\right)$ & $29 \pm 3$ & $29 \pm 3$ & 0.88 \\
Gestational age (weeks) & $39 \pm 1$ & $39 \pm 1$ & 0.57 \\
Gravidity & $2(2-3)$ & $2(1-3)$ & 0.71 \\
Parity & $1(1-1)$ & $1(0-1)$ & 0.42 \\
Baseline SBP (mm Hg) & $113 \pm 6$ & $117 \pm 10$ & 0.09 \\
\hline
\end{tabular}

Data presented as mean \pm SD or median (IQR). BMI: body mass index; SD: standard deviation; IQR: interquartile range; SBP: systolic blood pressure.

sors administered either PEI vs. PEB post-spinal anesthesia on MFI was assessed. A power calculation was performed for the difference between two dependent (pre- and post-spinal) and secondarily, two independent (PEI and PEB groups) means of MFI using data from our recently completed assessment of maternal microcirculation [15]. Using $\mathrm{G}^{*}$ Power (version 3.0.10, University of Dusseldorf) with a standard deviation of 0.2 , the calculated effect size is $1.472(10 \%$ change in capillary density) (alpha 0.05 , power 0.9 ). Eight (dependent) or 24 (independent) patients (12 per group) result in the appropriate power. The mean MFI values of all patients were compared before and after spinal anesthesia using paired Student's $t$-tests.

\section{Results}

Thirty-two participants were recruited and randomly assigned to either receive a PEI or PEB. Of these, 22 patients had complete video sets available for pre- and post-spinal analysis and of these, assigned to either receive a PEI $(n=11)$ or PEB if treatment was required $(\mathrm{n}=11)$.

Baseline characteristics were similar between the two groups, including preoperative hemodynamics (Table 1). Intraoperative characteristics are presented in Table 2. The mean dose of phenylephrine in the PEI group was $1,603 \pm 470 \mu \mathrm{g}$ with four patients who experienced hypotension requiring the rate of PEI to be increased. Hypotensive characteristics were identified if a patient's SBP decreases to $<90 \mathrm{~mm} \mathrm{Hg}$, or a decrease by $20 \%$ in SBP. Of those four, the infusion rate was increased prior to imaging in three patients, and after imaging in one patient. One patient received an additional $10 \mathrm{mg}$ ephedrine to treat hypotension. Nine patients in the PEB group received vasopressors during the 10 -min evaluation period with mean doses of $200 \pm 142 \mu \mathrm{g}$ of phenylephrine and $13 \pm 6$ mg of ephedrine.
The pre-spinal MFI $(2.78 \pm 0.27)$ was not significantly different than the post-spinal MFI $(2.65 \pm 0.34)$ for all participants (mean difference (MD): $0.13,95 \% \mathrm{CI}$ : -0.03 to $0.29, \mathrm{P}$ $=0.11$, Fig. 1). There were no significant differences between pre- and post-spinal MFI in the within-group comparisons (Fig. 2a (PEI) and b (PEB)). The between-group comparison of post-spinal MFI for the PEI group $(2.74 \pm 0.21)$ was not significantly different compared to the post-spinal PEB group $(2.56 \pm 0.42)$ (MD: $0.18,95 \% \mathrm{CI}:-0.11$ to $0.48, \mathrm{P}=0.22$, Fig. $3)$. There were no differences in total vessel density, perfused vessel density, or the proportion of perfused vessels between the PEI and PEB groups following administration of spinal anesthesia (Table 3).

Within the PEB group, seven participants received vasopressors prior to imaging, two participants received vasopressor after imaging and two did not receive any vasopressors. Subgroup analyses were conducted comparing the four subjects who did not receive vasopressors prior to imaging, to the seven subjects who did. This showed there was no significant difference between the mean MFI pre- (MD: $-0.06,95 \% \mathrm{CI}$ : -0.38 to $0.25, \mathrm{P}=0.66$ ) and post-spinal (MD: $0.17,95 \% \mathrm{CI}$ : -0.45 to $0.79, \mathrm{P}=0.56$ ).

\section{Discussion}

This study was designed to improve our understanding of how spinal anesthesia and phenylephrine influence maternal microcirculation. Our results showed no statistically significant difference in MFI after administration of spinal anesthesia (Fig. 2). This suggests that spinal anesthesia may have a limited impact on sublingual microcirculation. These findings are congruent with a recent report from our group showing that epidural analgesia may not impact sublingual microcirculation in laboring women [16].

Table 2. Intraoperative Characteristics

\begin{tabular}{llll} 
& Phenylephrine infusion $(\mathbf{n}=\mathbf{1 1})$ & Phenylephrine bolus $(\mathbf{n}=\mathbf{1 1})$ & P-value \\
\hline Duration of surgery $(\mathrm{min})$ & $53 \pm 27$ & $43 \pm 9$ & 0.15 \\
Total fluids given $(\mathrm{mL})$ & $1,559 \pm 412$ & $1,891 \pm 419$ & 0.08 \\
Estimated blood loss $(\mathrm{mL})$ & $586 \pm 158$ & $500 \pm 140$ & 0.19 \\
\hline
\end{tabular}

Data presented as mean \pm SD. SD: standard deviation. 


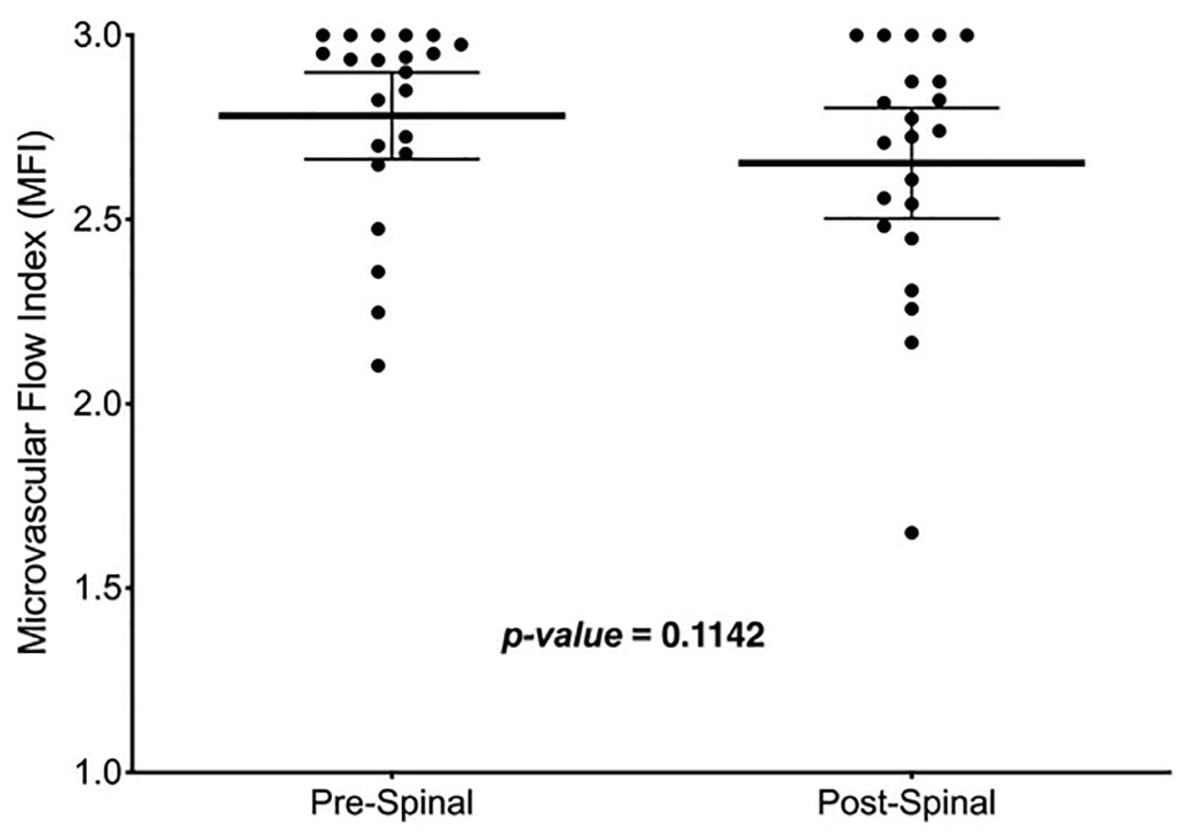

Figure 1. Mean MFI measurements pre- and post-spinal anesthesia entire cohort. Thick, solid line in the box represents the mean, whiskers represent the $95 \%$ confidence interval and solid dots are participants. MFI: microvascular flow index.

Prophylactic infusion of phenylephrine did not result in a statistically significant alteration of the microcirculation compared to those who only required PEBs to treat spinal anesthesia-induced hypotension (Fig. 2). Indeed, this is despite an average eight-fold greater dose of phenylephrine administered to the PEI group compared to the PEB group $(1,603 \mu \mathrm{g}$ vs. 200 $\mu \mathrm{g})$. Maier et al showed differences in sublingual MFI using SDF; however, they administered phenylephrine at an infusion rate of $1.4 \mu \mathrm{g} / \mathrm{kg} / \mathrm{min}$ with an average patient mass of $79.6 \mathrm{~kg}$ [17]. Therefore, this would be on average a three times greater dose than the baseline dose provided in the current study. Additionally, Maier et al titrated their dose to increase perfusion pressure by $20 \mathrm{~mm} \mathrm{Hg}$, whereas we have attempted only to prevent a $20 \%$ decrease in SBP [17].

Our inability to detect changes within the systemic vascular resistance and observe MFI differences pre- and post-spinal anesthesia may have been confounded by a lack of statistical power, the vasoconstrictive properties of phenylephrine and the clinical necessity to treat hypotension particularly in the PEB group. This inability is particularly evident in the planned pre- and post-spinal anesthesia comparison of the PEB group, to directly assess physiologic impact of spinal anesthesia where seven of 11 patients required support from vasopressors to maintain their blood pressure prior to SDF imaging. The timing and rate of vasopressor intervention in this group was necessary to ensure the safety and comfort of our patients throughout the course of our study protocol. Despite this clinical trial challenge, we did not adjust the study protocol to delay vasopressor administration or tolerate any more than a $20 \%$ decrease in SBP from baseline before treating appropriately.

While others have used periungal capillaries as a surrogate marker of capillary bed changes in the uterus and placenta in pregnant patients [18], we have previously suggested that sublingual microcirculation may be a useful indicator of those outcomes [15]. Our inability to detect any changes to MFI after administration of spinal anesthesia suggests that these observed hypotensive changes may not impact systemic microcirculation and therefore may not impact placental microcirculation. Similarly, we did not find any differences in sublingual MFI after prophylactic infusion of phenylephrine despite the direct alphaadrenergic agonism and expected vasoconstriction. Therefore, it must be considered that the amount of drug administered in the current study is simply below a physiologic threshold where microcirculatory changes are detectable by SDF. Accordingly, our results suggest that as much as $30 \mu \mathrm{g} / \mathrm{min}$ dose of phenylephrine does not have any strong influence on sublingual microcirculation and therefore placental blood flow.

An alternative explanation for the similar MFI measurements before and after the administration of spinal anesthesia is that the SDF probe is not sensitive enough to detect changes in MFI [9]. Given the hemodynamic perturbations that were observed after administration of spinal anesthesia, and that 13 of our 22 patients experienced hypotension, it is reasonable to assume that there were some alterations in the microvasculature; yet this trend did not correlate with MFI measured by the SDF probe.

Our group has previously shown no significant differences in MFI among pregnant women before and after administration of epidural analgesia, despite the potential for hypotension [16]. The next generation of microcirculatory microscope, incident dark field (IDF) imaging, includes technical improvements. However, despite improvements of IDF to detect total vessel density and proportion of perfused vessels, it has not been shown to have the same superior capacity to detect changes in MFI in transcutaneous microcirculatory measurements among infants [19]. 

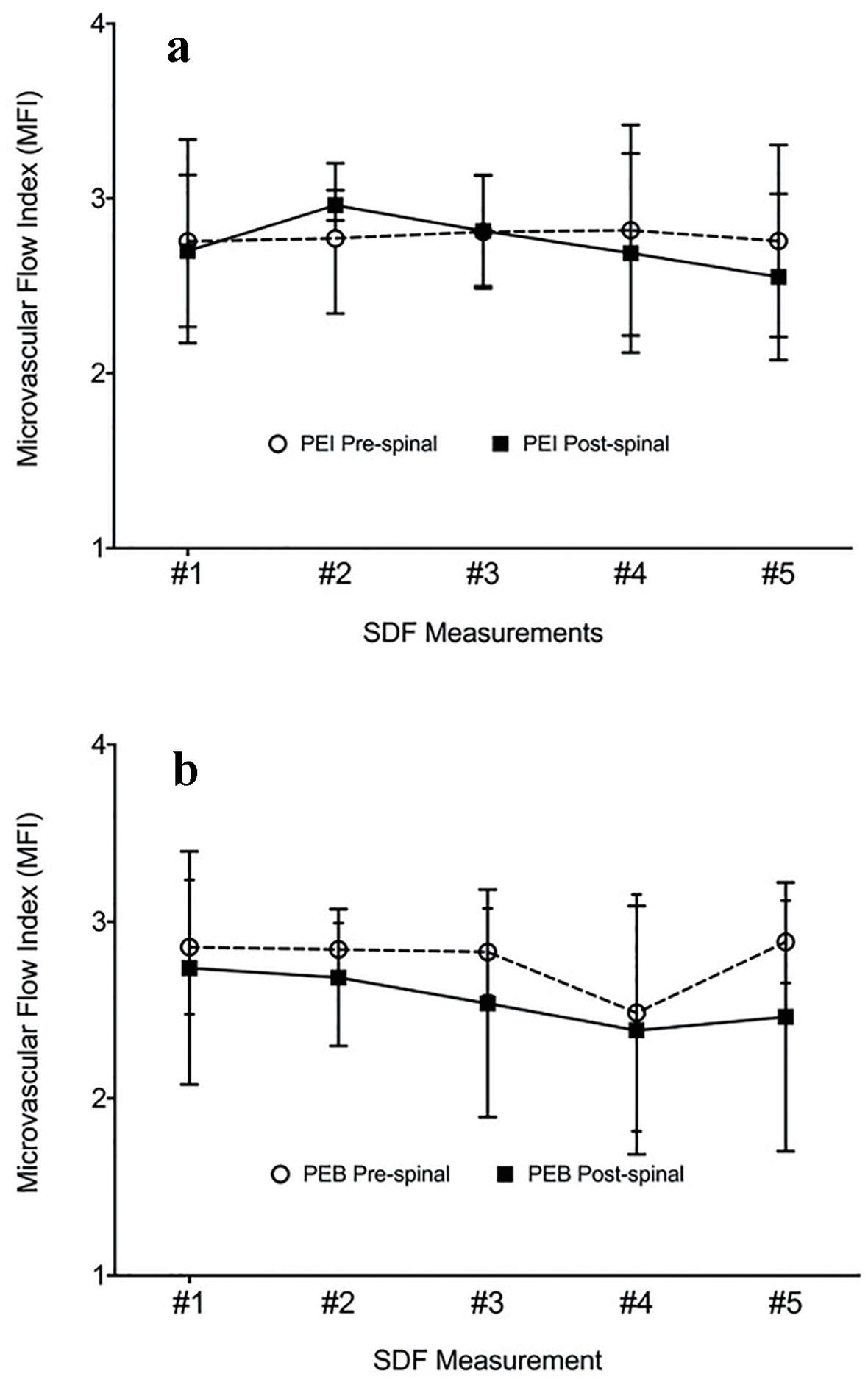

Figure 2. MFI measurements pre- and post-spinal anesthesia for the (a) phenylephrine infusion and (b) phenylephrine bolus groups.

Due to technical issues with SDF video quality, we were unable to capture the required 12 participants per group $(\mathrm{n}=$ 11) as determined a priori to detect a significant difference pre-post spinal anesthesia between PEI and PEB groups. The technical challenges included suboptimal imaging of the capillaries due to motion-induced image blurring by movement of the OPS device, the tissue and/or flowing red blood cells. Also, in larger vessels, especially during continuous flow, it is difficult to observe the granular nature of flowing blood cells due to blurring of images. These technical challenges introduce difficulties in measuring blood flow velocities in these vessels. For these reasons, recruitment was halted at 32 subjects. 


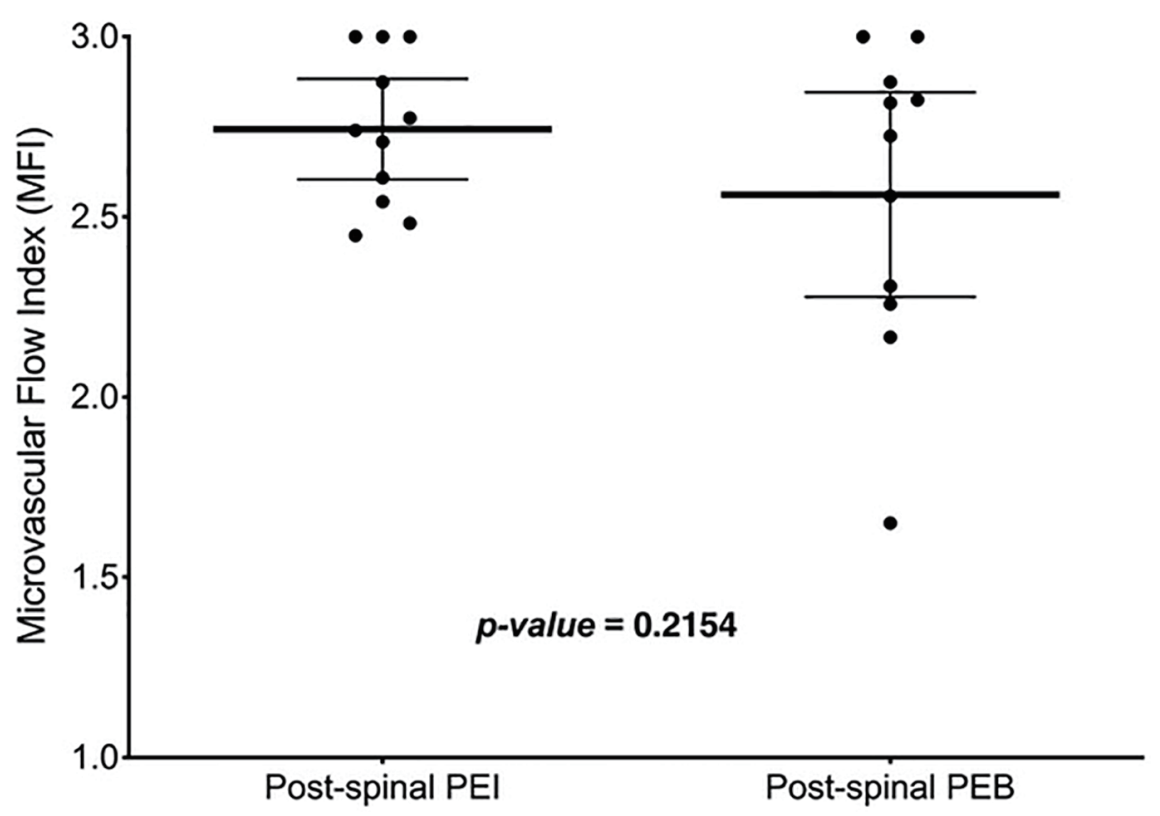

Figure 3. Post-spinal MFI between infusion and bolus groups was not significantly different $(P=0.22)$. Thick, solid line in the box represents the mean, whiskers represent the $95 \%$ confidence interval and solid dots are participants. MFI: microvascular flow index.

While providing important insight, this study was limited by its clinical nature and the necessity to treat hypotension for safety and symptomatic control. These necessary safety protocols limited out ability to control for the amount and timing of phenylephrine patients received and therefore it can be difficult to isolate the influence of spinal anesthesia on microcirculation when confounded by vasopressor. This influence should be further explored using a gravid animal model, where maintenance of normal blood pressure and symptom management is not a requirement, thus allowing a better comparison. Additionally, we have not attempted to measure the contribution of macrovascular changes in the response to spinal anesthesia or phenylephrine. The measurements may have helped us to correlate their influence with hemodynamic changes. Lastly, the hemoglobin level, which was not measured, appears to be a determinant of the capillary network and may impact the sublingual capillary density [20]. Future studies incorporating a positive control, such as a large vessel, and measuring hemoglobin levels may elucidate the influence of vasopressors or fluid resuscitation.

In conclusion, we have found no difference in MFI before or after administration of spinal anesthesia, nor between groups receiving phenylephrine as a prophylactic infusion or in required treatment boluses. These findings suggest that nei- ther spinal anesthesia nor PEI at $30 \mu \mathrm{g} / \mathrm{min}$ have a strong influence on microcirculatory blood flow. By extension, consideration of these practices likely does not pose a significant risk when providing regional anesthesia and hemodynamic support during cesarean delivery.

\section{Acknowledgments}

We would like to acknowledge the hard-working research staff of the Department of Women's \& Obstetric Anesthesia, without whom our programs of research would not be successful.

\section{Financial Disclosure}

This work was supported by a category B grant (\#12608) from the IWK Health Centre.

\section{Conflict of Interest}

The authors have no conflict of interest to disclose.

Table 3. Additional Post-Spinal Microcirculation Measures

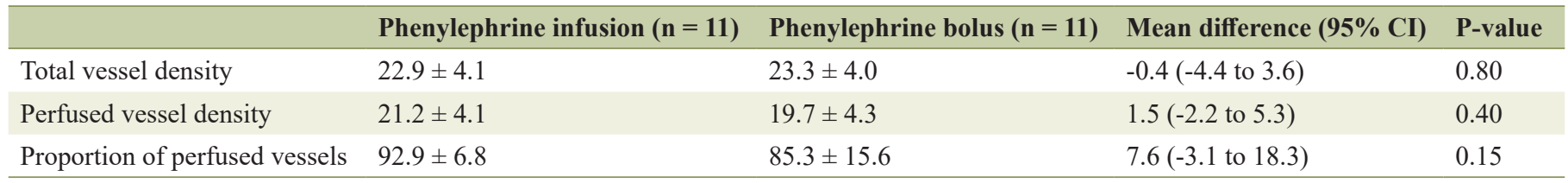

$\mathrm{Cl}$ : confidence interval. 


\section{Informed Consent}

All patients provided written informed consent prior to study participation.

\section{Author Contributions}

Ronald George contributed to the conception and design of the study, data analysis and writing of the manuscript. Colin Boyd contributed to the data collection, data analysis and writing of the manuscript. Dolores McKeen contributed to the design of the study, data interpretation and editing of the manuscript. Islam Saleh Abdo contributed to the design of the study, data interpretation and editing of the manuscript. Christian Lehmann contributed to the design of the study, data interpretation and editing of the manuscript.

\section{References}

1. Lee JE, George RB, Habib AS. Spinal-induced hypotension: Incidence, mechanisms, prophylaxis, and management: Summarizing 20 years of research. Best Pract Res Clin Anaesthesiol. 2017;31(1):57-68.

2. Habib AS. A review of the impact of phenylephrine administration on maternal hemodynamics and maternal and neonatal outcomes in women undergoing cesarean delivery under spinal anesthesia. Anesth Analg. 2012;114(2):377-390.

3. Allen TK, George RB, White WD, Muir HA, Habib AS. A double-blind, placebo-controlled trial of four fixed rate infusion regimens of phenylephrine for hemodynamic support during spinal anesthesia for cesarean delivery. Anesth Analg. 2010;111(5):1221-1229.

4. Cooper DW, Carpenter M, Mowbray P, Desira WR, Ryall DM, Kokri MS. Fetal and maternal effects of phenylephrine and ephedrine during spinal anesthesia for cesarean delivery. Anesthesiology. 2002;97(6):1582-1590.

5. Ngan Kee WD, Khaw KS, Lau TK, Ng FF, Chui K, Ng KL. Randomised double-blinded comparison of phenylephrine vs ephedrine for maintaining blood pressure during spinal anaesthesia for non-elective Caesarean section*. Anaesthesia. 2008;63(12):1319-1326.

6. Ngan Kee WD, Lee A, Khaw KS, Ng FF, Karmakar MK, Gin T. A randomized double-blinded comparison of phenylephrine and ephedrine infusion combinations to maintain blood pressure during spinal anesthesia for cesarean delivery: the effects on fetal acid-base status and hemodynamic control. Anesth Analg. 2008;107(4):1295-1302.

7. den Uil CA, Lagrand WK, Spronk PE, van der Ent M, Jewbali LS, Brugts JJ, Ince C, et al. Low-dose nitroglycerin improves microcirculation in hospitalized patients with acute heart failure. Eur J Heart Fail. 2009;11(4):386390.

8. Elbers PW, Ozdemir A, van Iterson M, van Dongen EP, Ince C. Microcirculatory imaging in cardiac anesthesia: ketanserin reduces blood pressure but not perfused capillary density. J Cardiothorac Vasc Anesth. 2009;23(1):95101.

9. Massey MJ, Shapiro NI. A guide to human in vivo microcirculatory flow image analysis. Crit Care. 2016;20:35.

10. Abdo I, George RB, Farrag M, Cerny V, Lehmann C. Microcirculation in pregnancy. Physiol Res. 2014;63(4):395408.

11. De Backer D, Creteur J, Dubois MJ, Sakr Y, Vincent JL. Microvascular alterations in patients with acute severe heart failure and cardiogenic shock. Am Heart J. 2004;147(1):91-99.

12. De Backer D, Creteur J, Preiser JC, Dubois MJ, Vincent $\mathrm{JL}$. Microvascular blood flow is altered in patients with sepsis. Am J Respir Crit Care Med. 2002;166(1):98-104.

13. Groner W, Winkelman JW, Harris AG, Ince C, Bouma GJ, Messmer K, Nadeau RG. Orthogonal polarization spectral imaging: a new method for study of the microcirculation. Nat Med. 1999;5(10):1209-1212.

14. De Backer D, Hollenberg S, Boerma C, Goedhart P, Buchele G, Ospina-Tascon G, et al. How to evaluate the microcirculation: report of a round table conference. Critical Care. 2007;11(5):101.

15. George RB, Munro A, Abdo I, McKeen DM, Lehmann C. An observational assessment of the sublingual microcirculation of pregnant and non-pregnant women. Int J Obstet Anesth. 2014;23(1):23-28.

16. George RB, DesRoches J, Abdo I, Lehmann C. Maternal microcirculation and sidestream dark field imaging: a prospective assessment of the association between labour pain and analgesia on the microcirculation of pregnant women. Clin Hemorheol Microcirc. 2015;60(4):389-395.

17. Maier S, Hasibeder WR, Hengl C, Pajk W, Schwarz B, Margreiter J, Ulmer H, et al. Effects of phenylephrine on the sublingual microcirculation during cardiopulmonary bypass. Br J Anaesth. 2009;102(4):485-491.

18. Ohlmann P, Jung F, Mrowietz C, Alt T, Alt S, Schmidt W. Peripheral microcirculation during pregnancy and in women with pregnancy induced hypertension. Clin Hemorheol Microcirc. 2001;24(3):183-191.

19. van Elteren HA, Ince C, Tibboel D, Reiss IK, de Jonge RC. Cutaneous microcirculation in preterm neonates: comparison between sidestream dark field (SDF) and incident dark field (IDF) imaging. J Clin Monit Comput. 2015;29(5):543-548.

20. Sharawy N, Hussein A, Hossny O, Refaa A, Saka A, Mukhtar A, Whynot S, et al. Effects of haemoglobin levels on the sublingual microcirculation in pregnant women. Clin Hemorheol Microcirc. 2016;64(2):205-212. 